\title{
Breaking the A chain: regulating mRNAs in development through CCR4 deadenylase Jocelyn Moore and Paul Lasko*
}

\author{
Address: Department of Biology and Developmental Biology Research Initiative, McGill University, 1205 Avenue Docteur Penfield, Montréal, \\ QC H3A 1B1, Canada \\ *Corresponding author: Paul Lasko (paul.lasko@mcgill.ca) \\ FI000 Biology Reports 2009, I:20 (doi: 10.3410/BI-20)
}

The electronic version of this article is the complete one and can be found at: http://FI000.com/Reports/Biology/content/I/20

\begin{abstract}
Post-transcriptional mechanisms of gene regulation have long been implicated in specifying embryonic pattern in many organisms. Experiments in Caenorhabditis elegans, Drosophila, and Xenopus have recently converged, pointing to the CCR4 deadenylase complex as a key effector that modulates the expression of proteins from specific germline mRNAs.
\end{abstract}

\section{Introduction and context}

A polarized distribution of maternally expressed proteins determines the spatial axes of the Caenorhabditis elegans and Drosophila embryos. Correct deployment of these proteins can entail asymmetric localization of their mRNAs, spatially restricted translational control, spatially restricted control of mRNA stability, or direct protein targeting. Often, two or more of these mechanisms operate redundantly. Recent advances in this area indicate that control of mRNA stability via poly(A) tail length is a key target for regulatory pathways essential to embryonic patterning.

\section{Major recent advances}

A key factor influencing mRNA stability and poly(A) tail length in Drosophila embryos is Smaug (Smg). Smg was first identified as a repressor of unlocalized nanos (nos) mRNA [1]. Smg negatively regulates nos translation by preventing recruitment of active cap-binding complex to the transcript [2], and it also targets nos mRNA for degradation by recruitment of the CCR4 deadenylase complex, a process blocked in the posterior pole plasm by Oskar [3]. Smg regulates degradation of unlocalized hsp83 mRNA in a similar fashion [4,5]. Influencing mRNA stability appears to be the more fundamental role of Smg. Expression profiling shows that mRNAs representing over half of the Drosophila genome (some 7,700 genes) are present in mature oocytes [6]. Of these, approximately 1,600 are degraded upon fertilization. In embryos lacking Smg, two-thirds of these mRNAs are stabilized. As these mRNAs are not enriched for the Smg recognition sequence, Smg many influence stability of these mRNAs in an indirect manner [6].

The CCR4 deadenylase complex is also recruited to target mRNAs by Bicaudal-C (Bic-C) [7] and by Nos itself [8]. Interestingly, these factors interact with different subunits of CCR4: Bic-C binds to the NOT3/5 subunit and Nos binds to NOT4. PUF-domain proteins, typified by Pumilio in Drosophila, represent another class of negative post-transcriptional regulators; such proteins recruit CCR4 through its POP2 subunit in yeast [9]. The direct interaction by which Smg recruits the CCR4 deadenylase is uncertain, although the NOT1 subunit was recovered in tandem affinity purification (TAP) experiments [4]. Surprisingly, CCR4 activity is also regulated by a cytoplasmic pool of a predominantly nuclear poly(A)binding protein, PABP2, a well-known stimulator of nuclear polyadenylation that with CCR4 targets certain cytoplasmic mRNAs for deadenylation during embryonic development [10]. The precise mechanism for this is unknown. 
CCR4-mediated deadenylation is counteracted by poly(A) polymerases and by Orb, the Drosophila homologue of cytoplasmic polyadenylation element-binding protein (CPEB), which promotes their recruitment. Interestingly, different poly(A) polymerases activate expression of specific germline mRNAs at different developmental stages. The poly(A) polymerase encoded by hiiragi ( $h r g$ ) is required during early stages of oogenesis, whereas that encoded by wispy (wisp, also known as Gld2) becomes essential at stage 10 of oogenesis and is required into early embryogenesis [11]. Wisp is required to extend the poly(A) tail of specific transcripts, such as bicoid, Toll, and torso, enabling their subsequent translation, and to stabilize oskar and nos RNAs in early embryogenesis [11,12].

The C. elegans germline determinant nos-2 is also under translational regulation; its expression is repressed in the somatic blastomeres of the early embryo [13]. nos-2 translational activation is the first known molecular event that is specific to the P4 primordial germ cell. Translational repression is maintained in somatic blastomeres by the interaction of four RNA-binding proteins (OMA-1, OMA-2, MEX-3, and SPN-4) with the nos-2 3' untranslated region (UTR) [14]. In the P4 cell, nos-2 translation becomes de-repressed when POS-1, another RNA-binding protein, outcompetes translational repressors for binding to the nos-2 $3^{\prime}$ UTR $[13,14]$. nos-2 mRNA is also targeted by degradation mechanisms in the somatic blastomeres, starting as early as the four-cell stage [13]. This is dependent on two highly similar zinc finger proteins, MEX-5 and MEX-6, and also involves the CCR4 deadenylase. When the NOT1 subunit of CCR4 is depleted by RNA interference, somatic processing bodies are decreased in number, nos-2 RNA is stabilized, and a green fluorescent protein reporter fused to the nos-2 $3^{\prime}$ UTR is translated in somatic blastomeres [15].

A similar CCR4-mediated mechanism of post-transcriptional regulation was implicated recently in vertebrate germline development as well. Xenopus $\mathrm{C} 3 \mathrm{H}-4$, a zinc finger protein closely related to POS- 1 , recruits CCR4 to shorten the poly(A) tails of target mRNAs that contain $\mathrm{A} / \mathrm{U}$-rich elements in their $3^{\prime}$ UTRs [16]. Key C3H-4 targets are emi1 and emi2, which encode proteins that inhibit the anaphasepromoting complex that is essential for progression beyond the metaphase I stage of meiosis. C3H-4 operates in opposition to $\mathrm{CPEB}$, and sequential cycles of polyadenylation and deadenylation of mRNAs encoding cell cycle regulators drive progression through meiosis [16].

Despite their sequence similarity, $\mathrm{C} 3 \mathrm{H}-4$ and POS- 1 have opposing regulatory effects: $\mathrm{C} 3 \mathrm{H}-4$ facilitates deadenylation whereas POS- 1 acts to counter translational repression. Also, Drosophila Bic-C promotes deadenylation or polyadenylation at different developmental stages, through associating with either CCR4 or Wisp $[7,11,12]$. Interactions between these RNA-binding proteins and different accessory proteins may explain these apparent contradictions; for example, RNA-binding proteins may regulate stability and activity of their targets by influencing whether they associate with processing bodies or germline P granules [15]. Although these particles share many components $[15,17]$, germline P granules lack the Sm-like (LSm) proteins LSm-1 and LSm-3, whose presence correlates with RNA degradation.

\section{Future directions}

Together, this work implicates CCR4 as a critical agent of post-transcriptional gene regulation of specific mRNAs underlying embryonic patterning. Future work will teach us more about the precise mechanisms by which CCR4 influences target mRNAs and whether its recruitment exclusively favors degradation or whether it can result in reversible translational silencing. Also, whereas some of the same RNA- and CCR4-binding proteins have been identified in developmental processes in both flies and worms, many thus far have been linked to this system in only one organism. It will be important to determine whether there is more commonality among CCR4interacting factors than is presently apparent or whether each system has developed distinct mechanisms to facilitate CCR4-mediated regulation.

\section{Abbreviations}

Bic-C, Bicaudal-C; CPEB, cytoplasmic polyadenylation element-binding protein; nos, nanos; Smg, Smaug; UTR, untranslated region; wisp, wispy.

\section{Competing interests}

The authors declare that they have no competing interests.

\section{Acknowledgements}

We are grateful to Martine Simonelig for helpful comments on the manuscript.

\section{References}

I. Smibert CA, Wilson JE, Kerr K, Macdonald PM: Smaug protein represses translation of unlocalized nanos $\mathrm{mRNA}$ in the Drosophila embryo. Genes Dev 1996, 10:2600-9.

2. Nelson MR, Leidal AM, Smibert CA: Drosophila Cup is an elF4Ebinding protein that functions in Smaug-mediated translational repression. EMBO J 2004, 23:150-9.

FI000 Factor 3.0 Recommended

Evaluated by Nancy Standart 9 Feb 2004

3. Zaessinger S, Busseau I, Simonelig M: Oskar allows nanos mRNA translation in Drosophila embryos by preventing its deadenylation by Smaug/CCR4. Development 2006, I33:4573-83.

4. Semotok JL, Cooperstock RL, Pinder BD, Vari HK, Lipshitz HD, Smibert CA: Smaug recruits the CCR4/POP2/NOT 
deadenylase complex to trigger maternal transcript localization in the early Drosophila embryo. Curr Biol 2005, I 5:284-94.

FI000 Factor 3.0 Recommended

Evaluated by Elmar Wahle 4 Mar 2005

5. Semotok JL, Luo H, Cooperstock RL, Karaiskakis A, Vari HK, Smibert CA, Lipshitz HD: Drosophila maternal Hsp83 mRNA destabilization is directed by multiple SMAUG recognition elements in the open reading frame. Mol Cell Biol 2008, 28:6757-72.

6. Tadros W, Goldman AL, Babak T, Menzies F, Vardy L, Orr-Weaver T, Hughes TR, Westwood JT, Smibert CA, Lipshitz HD: SMAUG is a major regulator of maternal mRNA destabilization in Drosophila and its translation is activated by the Pan Gu kinase. Dev Cell 2007, 1 2:143-55.

FI000 Factor 4.8 Must Read

Evaluated by Beat Suter 19 Jan 2007, Paul Lasko 16 Feb 2007

7. Chicoine J, Benoit P, Gamberi C, Paliouras M, Simonelig M, Lasko P: Bicaudal-C recruits CCR4-NOT deadenylase to target mRNAs and regulates oogenesis, cytoskeletal organization, and its own expression. Dev Cell 2007, I 3:691-704.

FI000 Factor 6.0 Must Read

Evaluated by Samuel Gunderson 4 Jul 2008

8. Kadyrova LY, Habara Y, Lee TH, Wharton RP: Translational control of maternal cyclin B mRNA by Nanos in the Drosophila germline. Development 2007, 134:1519-27.

9. Goldstrohm AC, Hook BA, Seay DJ, Wickens M: PUF proteins bind Pop2p to regulate messenger RNAs. Nat Struct Mol Biol 2006, 13:533-9.

FI000 Factor 3.3 Recommended

Evaluated by Mark Caddick 26 May 2006, Elmar Wahle 2 Jun 2006, Jeff Coller 5 Jun 2006
10. Benoit P, Mitou G, Chartier A, Temme C, Zaessinger S, Wähle E, Busseau I, Simonelig M: An essential cytoplasmic function for the nuclear poly(A) binding protein, PABP2, in poly(A) tail length control and early development in Drosophila. Dev Cell 2005, 9:5II-22.

II. Benoit P, Papin C, Kwak JE, Wickens M, Simonelig M: PAP- and GLD-2-type poly(A) polymerases are required sequentially in cytoplasmic polyadenylation and oogenesis in Drosophila. Development 2008, 135:1969-79.

12. Cui J, Sackton KL, Horner VL, Kumar KE, Wolfner MF: Wispy, the Drosophila homolog of GLD-2, is required during oogenesis and egg activation. Genetics 2008, 178:2017-29.

13. D'Agostino I, Merritt C, Chen P-L, Seydoux G, Subramaniam K: Translational repression restricts expression of the $C$. elegans Nanos homolog NOS-2 to the embryonic germline. Dev Biol 2006, 292:244-52.

14. Jadhav S, Rana M, Subramaniam K: Multiple maternal proteins coordinate to restrict the translation of $C$. elegans nanos-2 to primordial germ cells. Development 2008, 135:1803-12.

15. Gallo CM, Munro E, Rasoloson D, Merritt C, Seydoux G: Processing bodies and germ granules are distinct RNA granules that interact in C. elegans embryos. Dev Biol 2008, 323:76-87.

16. Belloc $E$, Méndez $R$ : A deadenylation negative feedback mechanism governs meiotic metaphase arrest. Nature 2008, 452:1017-21.

FI000 Factor 3.0 Recommended

Evaluated by Angel Nebreda 23 May 2008

17. Thomson T, Liu N, Arkov A, Lehmann R, Lasko P: Isolation of new polar granule components in Drosophila reveals $\mathbf{P}$ body and ER associated proteins. Mech Dev 2008, I25:865-73. 